\title{
Urban Touch
}

\author{
Edda Sickinger
}

Dieser Beitrag gibt einen Einblick in die tanzkünstlerische Forschung Urban Touch, die im Sommer 2018 an öffentlichen Orten Hamburgs umgesetzt wurde, um Berührungsprozesse im Stadtalltag zu ergründen. Sie wurde in der Affenfaust Galerie in Hamburg uraufgeführt. Die leitende Frage der Arbeit war es herauszufinden, welche Arten von Berührung unterschiedliche Orte einer Stadt anbieten und was passiert, wenn sich entgegen dieser verhalten wird. Durch choreografische Settings sollten die Berührungsdynamiken des Ortes aufgebrochen werden.

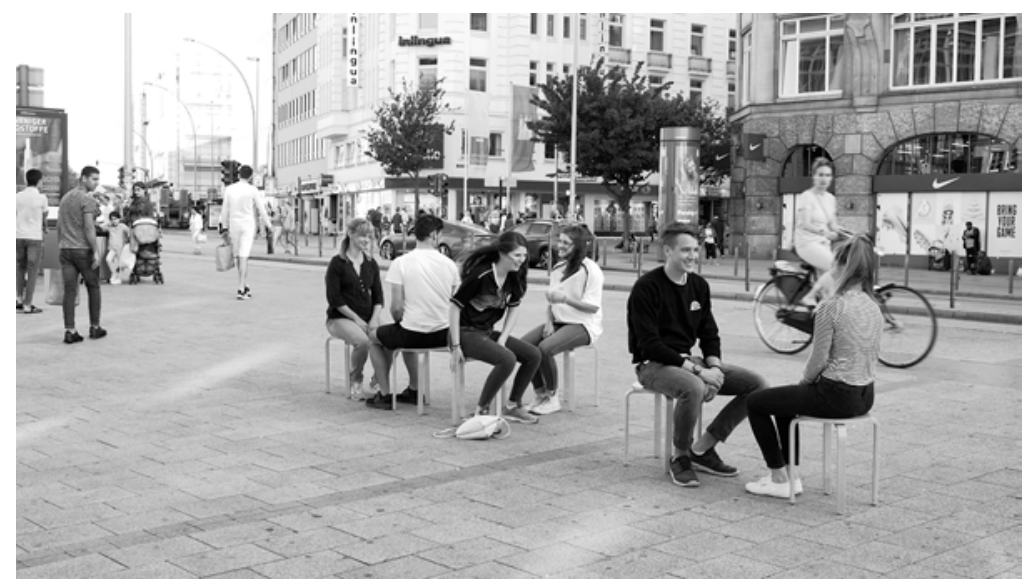

Abb. 1: Urban Touch, Performance Installation, Juli 2018 Guy Marsan, Edda Sickinger und Angela Kecinski aufdem Vorplatz des Hamburger Hauptbahnhofs gemeinsam mit Passant*innen, Videostill: Franz Sickinger, PicPacker.de. 


\section{Berührung ist Bewegung}

In Urban Touch haben sich Angela Kecinski, Guy Marsan und Edda Sickinger in die Dynamiken städtischer Beziehungen begeben, diese entdeckt und die Grenzen ausgetestet. In Anlehnung an Ideen $\mathrm{zu}$ empfindungsfähigen öffentlichen Räumen (Amin/Thrift 2016) sind Städte als fühlende, affektive und flüssige Umgebungen zu verstehen. Sie sind keine stabilen Zustände, sondern werden ständig durch menschliche und nicht-menschliche Bewohner*innen und Materie oft improvisiert hervorgebracht (Simone 2004).

Bezüglich ausgewählter städtischer Räume wurden in Urban Touch gewisse Erwartungshaltungen antizipiert, die Stadtbewohner*innen mit bestimmten Orten verbinden, um diese anschließend choreografisch zu brechen und sich gegensätzlich zu verhalten. Städtische Beziehungen wurden mit dem Fokus auf Berührungen betrachtet, indem die Performer*innen diese im Rahmen ihrer Aktionen beobachtend und intervenierend in den verschiedensten Formen wahrnahmen. Berührungen sollten hierbei nicht ausschließlich als Momente des physischen Kontakts verstanden werden, sondern als Prozess von Bewegungen der Annäherung und Distanzierung, sowohl physisch als auch in Affekt und Relation (Egert 2016). Erin Manning beschreibt dieses Phänomen als ein »reaching-toward« (Manning 2007: xiv).

Vor diesem Hintergrund wurde der Frage nachgegangen, wie sich die Aktionen und ihre Brechung einer wahrscheinlichen Erwartungshaltung auf die Berührungsprozesse vor Ort auswirken, insbesondere da Tanz im öffentlichen Raum Momente unkontrollierbarer Interaktionen schafft. Jay Pather (2013) nennt ein so beteiligtes informelles Publikum unvorhersehbar, da es sich situativ sowohl ablehnend als auch zugewandt zeigen kann.

\section{Intervention am Hauptbahnhof: Aushandeln, Spielen}

Der Bahnhof ist ein Ort, den zahlreiche Menschen aufsuchen, vorrangig, um zielgerichtet eine Reise anzutreten oder von dieser zurückkehren. Es herrscht hektischer Durchgangsbetrieb, meist mit Blick auf sich und die eingeschlagene Richtung.

Als choreografisches Setting wurden an einem Nachmittag sechs Hocker auf dem Vorplatz des Hauptbahnhofs platziert. Die drei Performer*innen setzten sich auf je einen Hocker und probierten unterschiedliche Sitzposi- 
tionen und -ausrichtungen aus. Die freien Hocker mit ihrem Aufforderungscharakter, sich hinzusetzen, erweckten die Aufmerksamkeit vieler Passant"innen (s. Abb. 1). Wenn jemand fragte, wie das Spiel funktionierte, wurde nur erklärt, dass jeder einen Zug hätte. Der Rest entschied sich im Handlungsverlauf. Pro Zug wurde der eigene Hocker neu im Raum positioniert und die Haltung auf diesem variiert, wodurch sich stets neue Relationen zu den Mitspielenden ergaben. Diverse Passant*innen blieben stehen und beobachteten das Spiel, kommentierten und unterstützten einzelne Entscheidungen. Einige fragten, was da gemacht würde und ob sie mitspielen dürften, wurden dann durch ein Winken eingeladen und stiegen mit ein.

Die Spieler*innen wägten ab, wie sehr sie mit ihrem Zug das Gesamtbild bestimmen durften. Einige passten sich mit ihren Zügen eher dem Bestehenden an, andere setzten neue Impulse. So änderte sich mit jedem Zug sowohl die räumlich-zeitliche Komposition als auch die Verbindungen und Relationen der Einzelnen untereinander. Die Mitspielenden konnten das Spiel jederzeit verlassen, wodurch der frei gewordene Hocker von einer anderen interessierten Person bespielt wurde. Das Spiel kam weitestgehend ohne verbale Absprachen aus. Nur zwischendurch, wenn zwei sehr nah mit direktem Augenkontakt beieinandersaßen, führte die unmittelbare Nähe zum Smalltalk. Viele Passanten“innen fotografierten. Trotz des Gegensatzes zum funktionalen Charakter der Bahnhofsumgebung ließen sich einige Passant"innen auf das spielerische Angebot ein und gestalteten die soziale Situation mit.

\section{Intervention auf der Reeperbahn: Stören, irritieren, reduzieren}

Die Reeperbahn ist ein bekannter Rotlichtbezirk und eine Party-Meile in Hamburg. Hier sind Hemmschwellen der Interaktion oft niedrig angesiedelt. Viele Menschen sind berauscht. Es war schwer abzusehen, was eine minimale reduzierte Aktion in dieser lauten Umgebung hervorrufen würde.

Angela Kecinski und Edda Sickinger positionierten sich am späten Abend inmitten der Fußgänger"innen auf der Reeperbahn, wandten sich einander zu und nahmen Blickkontakt miteinander auf. Dabei variierten sie den $\mathrm{Ab}$ stand zueinander, bewegten sich mit langsamen Schritten aufeinander zu und distanzierten sich wieder (s. Abb. 2). Sie zielten darauf ab, eine starke Verbindung und Intimität zueinander aufzubauen und diese trotz allem, 
was um sie herum passierte, aufrechtzuerhalten. Die leisen und langsamen Bewegungen und der intensive, teils über größere Distanz gehaltene Blickkontakt stand im Kontrast zur offenen, schrillen, schnellen Umgebung.

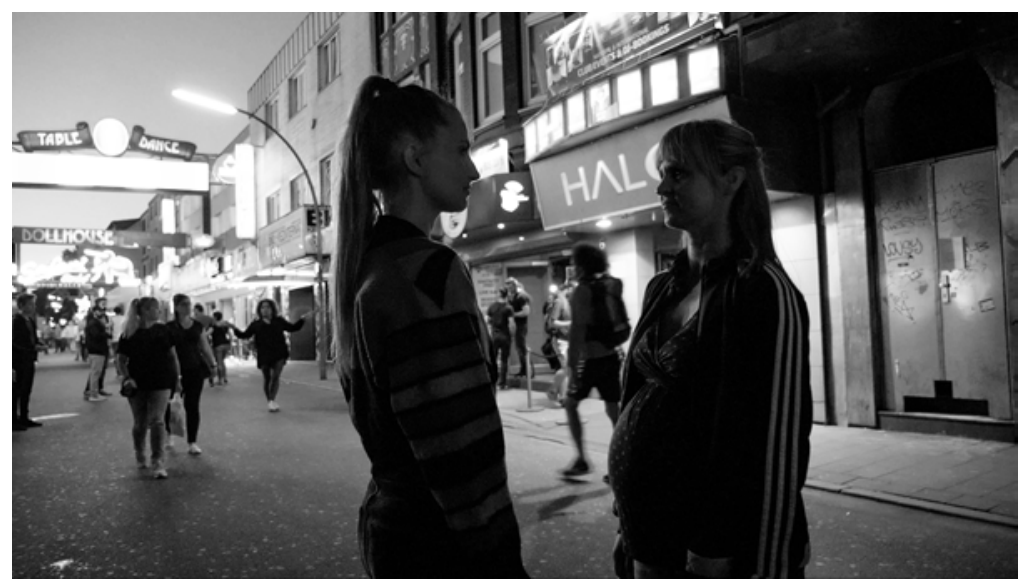

Abb. 2: Urban Touch, Performance Installation, Juli 2018 Edda Sickinger und Angela Kecinski aufder Reeperbahn in Hamburg, Videostill: Franz Sickinger, PicPacker.de.

Die Aktion provozierte verschiedenartige Reaktionen. Einige Passant"innen schauten den Performerinnen aus der Distanz unauffällig zu, andere bemerkten das als konträr intendierte Verhalten erst gar nicht. Wieder andere waren motivierter und sahen sich aufgefordert, selbst ins Geschehen einzugreifen. So nutzten einige Passant"innen den entstandenen Raum zwischen den Performer"innen als Bühne, um durch eigene Posen die Blicke auf sich zu ziehen. Andere wollten die Verbindung offensichtlich nicht unterbrechen, sodass sie die Performerinnen im großen Bogen passierten. Andere Passant"innen interagierten, indem sie sich ebenfalls einer Person zuwandten, den Blickkontakt aufnahmen und die räumliche Distanz zueinander variierten. Zwischendurch entstand ein Kreis aus mehreren Menschen, der sich im Wechsel weitete und verengte. Die Performer*innen wurden viel angeguckt, physisch näherte sich den beiden jedoch niemand. Manchmal blickten sie zurück, was den Blick der anderen Person meist wieder verscheuchte. 


\section{Intervention auf der Großen Freiheit: Sich entziehen}

Die Straße mit dem Namen Große Freiheit ist ebenfalls Teil des Reeperbahn Kiezes. Hier saß Guy Marsan bei Nacht 25 Minuten lang auf einem Wohnzimmerhocker mit geschlossenen Augen wie meditierend und bewegte sich nicht (s. Abb. 3). Was man von außen nicht sieht, ist, wie er sich auf seine Atmung konzentriert und mit seiner Wahrnehmung seinen Körper scannt, um trotz der sich überlappenden Songs aus den Clubs, der vielen Passant*innen und dem Lichtermeer, die Ruhe zu bewahren.

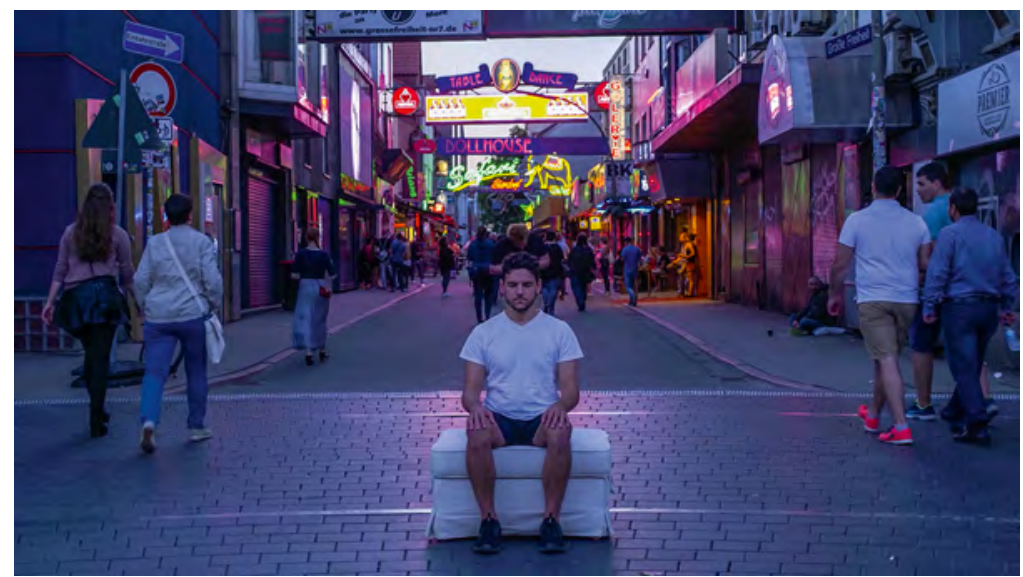

Abb. 3: Urban Touch, Performance Installation, Juli 2018 Guy Marsan aufder Großen Freiheit in Hamburg, Videostill: Franz Sickinger, PicPacker.de.

Diese Aktion erregte besonders viel Aufmerksamkeit. Körperlich näherte sich dem Performer niemand, mit Ausnahme einer Person, die versehentlich gegen ihn stolperte. Aus Gesprächen war zu entnehmen, wie sich Passant*innen mit dem Möglichkeitsspielraum auseinandersetzten, wie sie interagieren könnten, z.B. sich dazu setzen, was schließlich aber nicht getan wurde. Viele bemerkten die Kamera und sprachen das Team darauf an, was die Aktion aussagen wollte. Viele mutmaßten, dass mit der Idee gespielt würde, welchen Reizen Menschen im Alltag ausgesetzt seien und was alles auf sie einprasselte. Sie spekulierten über mögliche Botschaften. Ist es ein Statement, ein Hungerstreik, hebt er gleich ab? Ist das eine Puppe? 


\section{Beobachtungen aus den Interventionen}

Die Aktionen lösten an den jeweiligen Orten ihres Auftretens unterschiedliche Berührungsprozesse aus, die einen Bruch mit den alltäglichen städtischen Dynamiken erkennen ließen. Im Rahmen der Intervention am Hauptbahnhof wurde der spielerische Charakter für das Publikum durch das Vorhandensein und die Anordnung der Sitzmöglichkeiten auf offenem Platz als Bruch mit dem Alltäglichen schnell ersichtlich. Hierdurch fühlten sich viele zur Teilnahme eingeladen und nutzten diese Gelegenheit.

Im Rahmen des Spiels wurde sowohl Raum für kleine zwischenmenschliche Gesten geschaffen als auch die Aufmerksamkeit für die Kommunikationsfähigkeit des eigenen Körpers innerhalb der Gruppe geschärft. Die Berührungen zeigten sich vor allem in einer Art Aushandlung dessen, wie sich die einzelnen Spielteilnehmer*innen in der räumlichzeitlichen Komposition stets neu positionierten und welche neuen Relationen und Impulse sie durch die Wahl ihrer Lokation, der Sitzhaltung, der körperlichen Ausrichtung den Mitspielenden gegenüber und der Kommunikationsweise im Spiel setzten. Die Intervention hat dem sonst eher funktionalen Ort, der in der Regel zielgerichtet aufgesucht wird, die abweichende Komponente des nicht zielgerichteten, spielerischen Aushandelns hinzugefügt. Der freie und improvisierte Spielcharakter erlaubte es allen Beteiligten, je nach Befinden zu agieren und so war sowohl forsches, abwägendes als auch zögerliches Verhalten zu beobachten. Temporär entstanden auch herausfordernde Momente, beispielsweise wenn sich zwei Spieler*innen mit direktem Augenkontakt gegenübersitzend wiederfanden und die vermutlich als unangenehm empfundene direkte körperliche Nähe mit Humor und einem Gespräch gelockert wurde, bis sich eine der involvierten Personen mit dem nächsten Zug einen neuen Platz suchen konnte. Im Verlauf des Spiels ließ sich nicht mehr ausmachen, wer die Aktion initiiert hatte und wer spontan mit eingestiegen war. Der Bruch mit der Dynamik des Ortes zeigte sich zudem an den zahlreichen Zuschauenden, die das Miterlebte fotografierten.

Das Blick-Duett auf der Reeperbahn musste in der lauten und schnellen Umgebung erst einmal entdeckt werden. Die reduzierten Handlungen der Performerinnen schufen einen umso breiteren Möglichkeitsspielraum für Handlungen seitens der Passant*innen, der auch genutzt wurde. Die Performerinnen begaben sich mit ihrem reduzierten, aber präsenten Auftreten 
in eine vulnerable Situation und waren auf diverse Reaktionen gefasst. Die Passant*innen schienen von dieser Aktion auf verschiedene Art und Weise berührt zu sein und trugen dazu bei, neue Relationen herzustellen. Einige waren vermutlich aufgrund ihrer Erwartungshaltung nicht auf Art und Intensität der Berührung, mit der sie sich konfrontiert sahen, gefasst. Die Beteiligten mussten sich spontan innerhalb der Situation beispielsweise mit ihrer Überforderung, Neugier oder schlicht Überraschung arrangieren. Manche Reaktionen, vor allem die Posen, wirkten brutal in der Dominanz ihres Eingreifens. Temporär ergab sich eine Ansammlung von Menschen, die eine gemeinsam hervorgebrachte Choreografie aus Blicken, Annäherungen und Distanzierung zeigte.

Im Gegensatz zum Duett schien die öffentliche Meditation in sich geschlossen und wenig Raum für Kommunikation zu bieten. Durch die innere Erfahrung des Performers in Bezug auf die spezielle Situation und die größtenteils distanzierten, aber interessierten Reaktionen der Leute, verband die Beteiligten eine weniger direkte Kommunikation des voneinander Berührtseins. Die Berührung mag im Einfühlen, der Ablehnung, der (fehlenden) Identifikation oder dem Ignorieren des Wahrgenommenen liegen. Die Passant"innen verstanden sofort, dass die Handlung inszeniert war und erkannten sie als Kunstaktion. Die Meditation auf der Reeperbahn wirkte wie ein Statement und veranlasste Passant*innen zu Deutungen, jedoch nicht zur unmittelbaren eigenen Teilhabe. Die Möglichkeiten, sich beispielsweise zum Performer zu setzen, wurde von den Zuschauenden nur theoretisch durchgespielt. Spekuliert werden könnte über die Frage, welchen Einfluss Gender auf die entstehenden Dynamiken vor allem während der beiden Aktionen auf der Reeperbahn ausübte.

Die choreografischen Settings zielten darauf ab, die antizipierten Berührungsdynamiken der jeweiligen Orte aufzubrechen. Die minimalen Interventionen haben diese temporär verändert, indem die Wahrnehmung der Beteiligten auf intime Kommunikationen gelenkt wurde. Die Akteure wurden sich ihrer selbst, ihrer Mitmenschen und den Relationen des Berührens bewusst, wodurch neuen Handlungsmöglichkeiten und Erfahrungsräume entstanden, in denen Kommunikation situativ und improvisiert ausgehandelt wurde. Welche Rolle sie in dieser einnehmen wollen, entschieden sie selbst, was offensichtlich auch durch die jeweilige Persönlichkeit der Beteiligten bestimmt ist. 
Es ist davon auszugehen, dass die Reaktionen nicht allein von den kontrastierenden Bewegungen und der körperlichen Präsenz der Performer*innen ausgelöst wurden. Einige Reaktionen waren sicherlich darauf zurückzuführen, dass die Passant"innen die Aktionen in ihrer Funktion als Kunst erkannt haben, zusätzlich verstärkt durch die Präsenz der Kamera. Einige Settings waren klarer als Kunstaktionen identifizierbar als andere, was die Reaktionen der Passant*innen zeigten. Dennoch haben die Aktionen die Dynamiken des Ortes temporär neugestaltet, auch mit dem Wissen, dass es sich um inszenierte Kunstaktionen handelte. Möglicherweise sogar genau deswegen.

Die choreografischen Interventionen von Urban Touch haben gezeigt, wie Akteure einer Stadt in und mit dieser improvisieren und in ständiger Bewegung und Aushandlung sind. Es wurde der Spielraum der einzelnen Personen offenbart, soziale Dynamiken zu gestalten. Unter anderem sind hiermit auch die Unvorhersehbarkeit und Unkontrollierbarkeit der Aktionen im dynamischen öffentlichen Raum zu begründen. Es zeigte die Vulnerabilität der Körper innerhalb einer fordernden Umgebung, aber auch die Wirksamkeit, die sozialen Dynamiken eines Ortes mitzugestalten.

\section{Literatur}

Amin, Ash/Thrift, Nigel (2017): Seeing Like a City, Cambridge: Polity Press.

Egert, Gerko (2016): Berührungen. Bewegung, Relation und Affekt im Zeitgenössischen Tanz, Bielefeld: transcript.

Manning, Erin (2007): Politics of Touch: Sense, Movement, Sovereignty, Minneapolis, MN: University of Minnesota Press.

Pather, Jay (2013): Shifting spaces, tilting time, in: Edgar Pieterse/AbdouMaliq Simone (Hg.), Rogue Urbanism - Emergent African Cities, Johannesburg: Jacana Media/African Centre for Cities, S. 433-443.

Simone, AbdouMaliq (2004): People as Infrastructure: Intersecting Fragments in Johannesburg, in: Public Culture, Jg. 16 H. 3, S. 407-429. 